\title{
Diagnóstico DE ARCHIVos MUNiCipales DE LA ANTIGUA PROVINCIA ZOQUE DEL ESTADO DE CHIAPAS*
}

\author{
Henry Moguel Villatoro**
}

\section{Resumen}

En 2012 la Universidad de Ciencias y Artes de Chiapas (Unicach) en conjunto con la asociación civil Apoyo al Desarrollo de Archivos y Bibliotecas de México (ADABI) llevaron a cabo el proyecto "Rescate de archivos históricos de la provincia zoque (siglos XVI al XXI)", con el objetivo de hacer un diagnóstico de los archivos de 25 municipios del estado de Chiapas, los cuales durante la época colonial formaron parte del territorio de la antigua provincia zoque de la alcaldía mayor de Chiapa. Como resultado de éste diagnóstico se detectaron tres archivos de ayuntamientos, siete archivos parroquiales y 16 archivos del registro civil, que en su mayoría conservan documentos de los siglos XIX y XX y en menor cantidad de los siglos XVII y XVIII.

Palabras clave: Rescate de archivos, historia de Chiapas, provincia zoque.

\section{Abstract}

In 2012 the University of Science and Arts of Chiapas and the association Development of Archives and Libraries of Mexico developed the project "Recover of historical archives of the zoque province (xVI to XXI centuries)", with the aim of make a diagnosis of archives from 25 municipalities of Chiapas, that in the colonial era were part of ter-

* La información de los archivos presentados en este diagnóstico fueron recopilados por Nabor Chandoquí, Levi Morales, Belsaida Morales y Josué Santiago, egresados de la licenciatura en historia de la Universidad de Ciencias y Artes de Chiapas (Unicach), asimismo se contó con la colaboración científica de los investigadores Sergio Nicolás Gutiérrez, Fermín Ledesma y Justus Fenner; además del apoyo logístico de Óscar Janiere Martínez, Fabiola Aguilar y Víctor Gallardo; y la asistencia económica de Apoyo al Desarrollo de Archivos y Bibliotecas de México (ADABI A.C.).

** Archivo Histórico de la Universidad de Ciencias y Artes de Chiapas. 
ritory of the zoque province at the mayoralty of Chiapa. As a result of this diagnosis were detected three archives from local councils, seven archives of parishes and 16 archives from vital record, which mostly have documents from the nineteenth and twentieth centuries and and to a lesser extent of the seventeenth and eighteenth centuries.

Keywords: Rescue archives, Chiapas history, zoque province.

\section{La provincia zoque durante la colonia}

Durante la época colonial la alcaldía mayor de Chiapa cubría parte del actual estado de Chiapas y se encontraba dividida en diversas provincias, entre ellas destacaba la provincia compuesta por pueblos indios hablantes de una antigua lengua nombrada por los españoles como la de los zoques, que desde tiempos prehispánicos se encontraba extendida desde la costa del pacífico y el lado oeste del territorio chiapaneco, hasta el extremo sureste de Veracruz y el extremo oeste del estado de Tabasco, manteniendo una estrecha relación lingüística con los hablantes de las lenguas mixe y popoluca, además de estar vinculados históricamente con la primera civilización urbana de Mesoamérica: la cultura olmeca. ${ }^{1}$

El régimen colonial también concentró población hablante de la lengua zoque en la provincia del Priorato de Chiapa y la del valle de Jiquipilas, por lo tanto el territorio de los pueblos zoques en esta época se extendió en diversas subregiones geográficas con distintas características ecológicas, ubicadas en la parte oeste del estado de Chiapas, que de norte a sur se localizan en el siguiente orden: llanuras del Golfo, estribaciones de Chapultenango, sierra de Tapalapa, sierra de Comeapa, sierra de Tecpatán, valles de Jitotol, valles de Tuxtla y Ocozocoautla y los valles de Jiquipilas y Cintalapa. ${ }^{2}$

1 Breves resúmenes con referencias a los estudios realizados sobre esta población durante el siglo xx los ofrecen Lee, "La lingüística histórica y la arqueología"; Thomas, "Los zoques"; y Lisbona, En tierra zoque; además del primer estudio colectivo sobre esta población de Villa Rojas, et al., Los zoques de Chiapas.

2 Las referencias a las subregiones y a la ubicación de los pueblos zoques durante la época colonial que se mencionan en este trabajo se obtuvieron de los estudios de Viqueira, Indios rebeldes e idólatras, y "Auge y decadencia de las montañas zoques". 


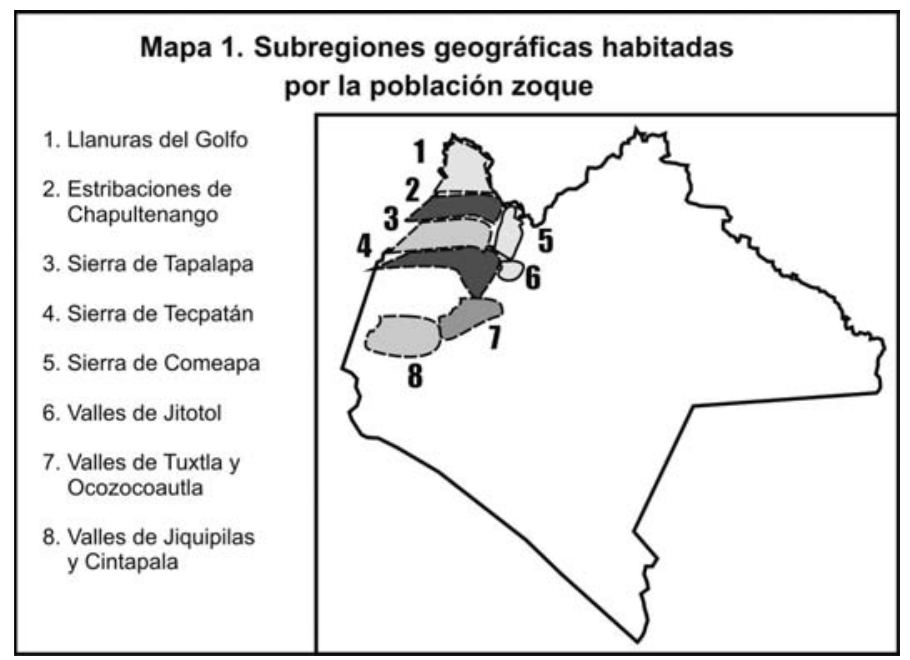

La provincia de los zoques con tierras calientes y frías propicias para el cultivo de cacao y la recolección de grana silvestre respectivamente, abarcaba los pueblos de: Sunuapa, Ixtapangajoya, Ixtacomitán, Solosuchiapa, Amatán, Ostuacán, Nicapa, Sayula, Cuscahuatán, Coalpitán, Chapultenango, Ixhuatán, Tapilula, Comistahuacán, Pantepec, Solistahuacán, Comeapa, Jitotol, Ocotepec, Tapalapa, Coapilla, Tecpatán, Chicoacán, Quechula, Chicoasén y Osumacinta. Mientras que el Priorato de Chiapa, con tierras húmedas aptas para la agricultura y la ganadería, sólo lo administraba el pueblo zoque de Tuxtla junto a otros seis pueblos indios hablantes de la lengua chiapa o chiapaneca. Dentro de la provincia del valle de Jiquipilas, donde se establecieron estancias de ganado y cultivos de caña de azúcar, se ubicaban los pequeños poblados de Magdalena de la Pita, Tacuasintepec, Cintalapa, Jiquipilas y Ocozocoautla.

El auge de la provincia zoque a finales del siglo XVI y principios del XVII se debió principalmente a la fabricación de textiles en los pueblos de la sierra de Tecpatán, a la recolección de grana silvestre en la de sierra de Tapalapa y a la producción de cacao en las estribaciones de Chapultenango. Estas mercancías eran embarcadas en el puerto fluvial de Quechula para ser llevadas por el río Grijalva hasta las costas de Tabasco, o también eran concentradas en la población de Chiapa de Indios para enviarlas a otras 


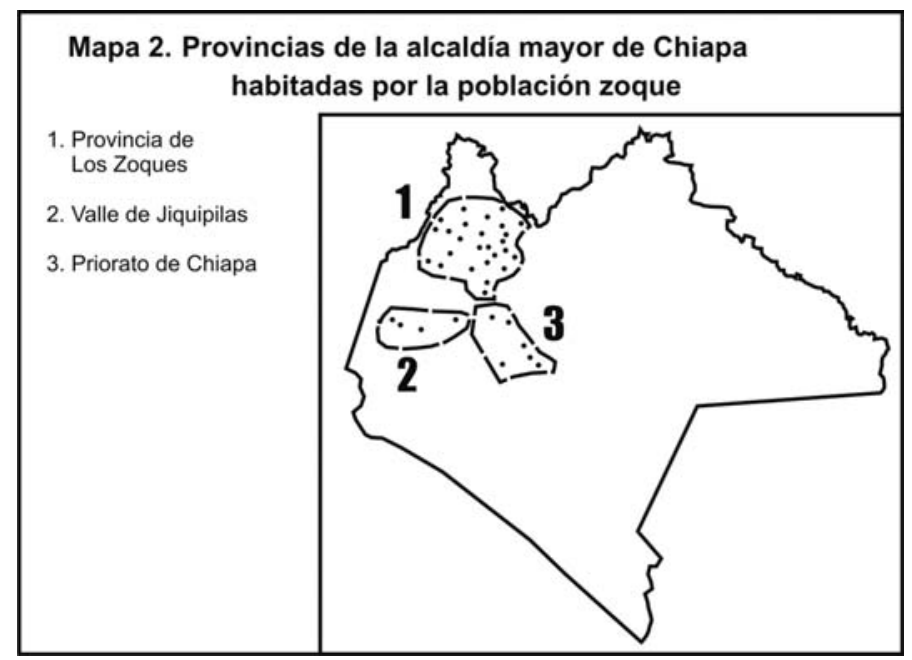

regiones de la alcaldía mayor de Chiapa. A principios del siglo XVIII, la prosperidad de la provincia zoque decayó debido a la brutal disminución de su población a causa de las epidemias y la reducción de los poblados, por lo cual esta provincia quedó convertida en un conjunto de pequeñas aldeas, a excepción de los pueblos de Tecpatán, Quechula y Copainalá que continuaron con una mayor atención de los frailes dominicos. ${ }^{3}$

En los poblados de los valles de Jiquipilas y Cintalapa, fundados por los dominicos pero administrados por el clero secular, es muy probable que la población zoque se encontrara reducida a la llegada de los españoles, ya que a fines del siglo XVI, los pueblos de Jiquipilas, Tacuasintepec y Santa María Magdalena de la Pita, fueron abandonados por sus habitantes para habitar el despoblado istmo de Tehuantepec o conducirse hacia los pueblos de Ocozocoautla y Tuxtla, los cuales mantienen durante los siglos XVII y XVIII un crecimiento demográfico mayor al del resto de los pueblos de la Depresión central de Chiapas. La desaparición de diversos pueblos en esta región, podría sugerir que la población zoque haya prácticamente desaparecido en la tercera década del siglo XVIII, por lo tanto estancias y fincas hicieron uso de mano de obra traída del continente africano. ${ }^{4}$

3 Datos obtenidos a partir del estudio elaborado por: Viqueira, "Auge y decadencia...", pp. 394-412. 
La muestra más notoria de los cambios en la organización social de los pueblos zoques durante la época colonial, se manifestó a través de la construcción de parroquias y conventos dominicos en los pueblos de la provincia zoque, destacando el gran convento prioral de Tecpatán, considerado el centro político y religioso de esta provincia, y el segundo pueblo más importante de la alcaldía mayor de Chiapa. Los dominicos establecieron en Tecpatán la sede de su priorato o convento formal con el apoyo de las vicarías de Copainalá, Tapalapa, Jitotol, Ixtacomitán y Chapultenango. Además de ser el único priorato en esta provincia, Tecpatán destacaba entre los demás conventos dominicos establecidos en el territorio chiapaneco, por ser el que mayor número de pueblos tuvo bajo su jurisdicción, por encima de los administrados por los conventos de Chiapa de Indios, Ciudad Real, Comitán, Copanaguastla y Ocosingo. ${ }^{5}$

El final de la época colonial no significó la suspensión de la vida cotidiana de los pueblos zoques, aunque diezmados por las enfermedades y las demandas económicas impuestas por las autoridades españolas, continuaron con su crecimiento y expansión en territorios de difícil acceso, evitando así conflictos o disputas con las nuevas élites políticas o hacendados de la región, aunque hasta ahora no se tengan datos de su situación durante el siglo XIX, es posible que hayan requerido de la intervención de las autoridades y de la participación política en defensa de sus intereses ante las políticas del gobierno mexicano al cual quedó federado el estado de Chiapas a partir de $1824{ }^{6}$

4 Cambios demográficos analizados estadísticamente por: Viqueira, Indios rebeldes..., pp. 1931. También durante esta época los habitantes de origen zoque de la región de Jiquipilas continuaron con su religiosidad prehispánica a través de ritos practicados en cuevas del cañón del río la venta, como lo demuestra el estudio etnohistórico de: Aramoni, Los refugios de lo sagrado.

5 La importancia y las características del convento de Tecpatán son descritas detalladamente por: Guzmán, "Santo Domingo de Tecpatán”, pp. 32-37.

6 Los últimos estudios de corte histórico y antropológico de: Ortiz, Lengua e historia entre los zoques; y de Lisbona, Sacrificio y castigo entre los zoques, sólo presentan algunas referencias sobre la última década del siglo XIX. 


\section{La falta de estudios históricos sobre los zoques y la desaparición de los archivos}

Durante el siglo xx, la población zoque en comparación con la población maya, ha merecido menor atención por parte de los científicos sociales, quienes han sido atraídos por los descubrimientos arqueológicos, la promoción turística internacional y el levantamiento armado del Ejército Zapatista de Liberación Nacional (EZLN) ${ }^{7}$ en 1994. Los primeros estudios acerca de la población zoque se realizaron durante la década de 1960, a causa de las afectaciones originadas por la construcción de las presas Malpaso, Chicoasén y Peñitas en el cauce del río Grijalva; en la década de 1970 por los descubrimientos y la explotación de yacimientos petrolíferos en la planicie costera de Tabasco; y sobre todo a partir de una catástrofe natural, la erupción del volcán Chichonal en 1982, que afectó a poblaciones zoques obligándolas a emigrar a diversos municipios del estado y del país. ${ }^{8}$

Actualmente se registran 53 mil 839 hablantes de la lengua zoque en la entidad, concentrados principalmente en un área conformada por 14 municipios: Chapultenango, Copainalá, Francisco León, Ixhuatán, Ixtacomitán, Jitotol, Juárez, Ocotepec, Ostuacán, Pantepec, Rayón, Tapalapa, Tapilula y Tecpatán. En 15 municipios aunque se registran menor número de hablantes, se consideran dentro de la región histórica de los zoques, como: Amatán, Berriozábal, Chicoasén, Cintalapa, Coapilla, Ixtapangajoya, Jiquipilas, Ocozocoautla de Espinosa, Osumacinta, Pichucalco, Pueblo Nuevo Solistahuacán, San Fernando, Solosuchiapa, Sunuapa y la capital del estado Tuxtla Gutiérrez. Tras la erupción del volcán Chichonal también se consideran las poblaciones reubicadas en cuatro municipios fuera del área históricamente zoque como: Acala, Chiapa de Corzo, Ocosingo y Villaflores. ${ }^{?}$

Aunque en los últimos años se han realizado diversos estudios sobre

7 Sobre el olvido de los zoques y los proceso ocurridos paralelamente al movimiento armado del EZLN en las distintas regiones habitadas por esta población, véase: Lisbona, "Olvidados del neozapatismo: los zoques chiapanecos".

8 Los estudios más recientes sobre la población zoque de Chiapas se han enfocado mayormente al impacto de la erupción del volcán Chichonal, véase: Laureano Reyes, Los zoques del volcán; Enrique Hidalgo, La fiesta del enojo; y Marina Alonso, Los zoques bajo el volcán. 9 En la consulta en línea de los tabulados básicos del Censo de Población del INEGI del 2010 solo aparece la población hablante de lenguas indígenas de la entidad, mientras que en los 


\section{Mapa 3. Municipios habitados por la población zoque}

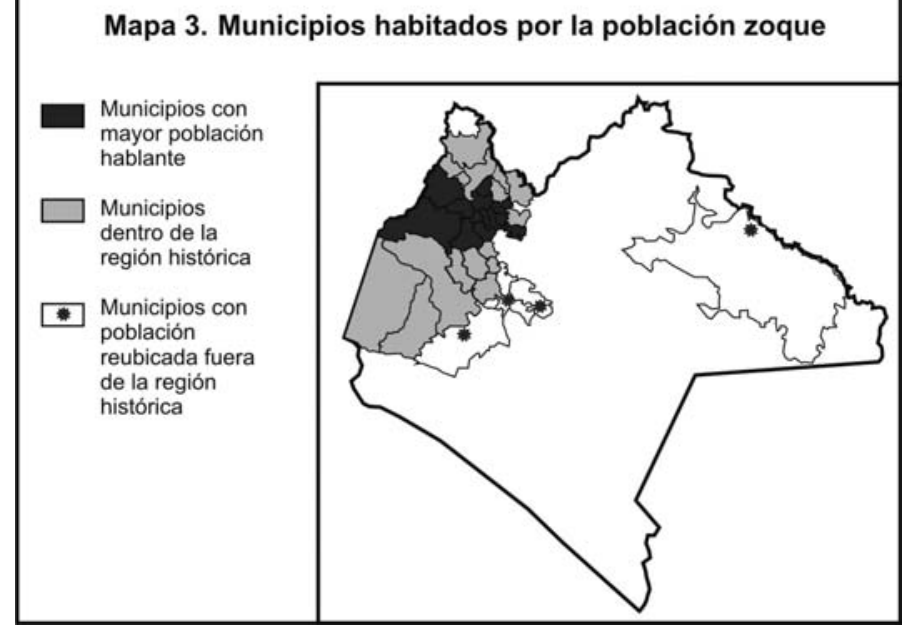

la población zoque de Chiapas, se desconoce gran parte de su historia moderna y contemporánea, la falta de investigaciones históricas obedece principalmente a la carencia de fuentes de información y a diversas situaciones que padecen los archivos históricos en el estado de Chiapas, las cuales van desde las restricciones en su acceso, el deplorable estado de conservación de los documentos hasta la destrucción intencional de los archivos; como lo ocurrido en abril de 2011 con el archivo del ayuntamiento de Copainalá, el cual fue quemado casi en su totalidad justo antes de iniciar el programa de rescate programado por la Dirección de Patrimonio e Investigación Cultural del Consejo Estatal para las Culturas y las Artes de Chiapas (Coneculta). ${ }^{10}$

Además de la negligencia y la irresponsabilidad de las autoridades municipales, los archivos de los ayuntamientos se encuentran en riesgo por

del Conteo de Población del 2005 aparece la población hablante de lenguas indígenas de cada municipio.

10 Durante más de un año Óscar Janiere Martínez Ruiz trabajó en la búsqueda del archivo del ayuntamiento de Copainalá hallado en la bodega de la agencia municipal del ejido Nueva Mezcalapa, a través de sus gestiones el cabildo aprobó el traslado del archivo a Tuxtla Gutiérrez para poder organizarlo y clasificarlo, desafortunadamente el día que realizarían el traslado fue llevado al tiradero de basura y quemado por el encargado de la bodega con la anuencia de una autoridad municipal. Este archivo también contaba con documentos del siglo XIX del antiguo Departamento de Mezcalapa que abarcaba diez ayuntamientos. 
el desinterés de las autoridades como fuente para garantizar la transparencia y el acceso a la información aunado a la falta de recursos para su rescate y conservación. A pesar de los esfuerzos y el interés de algunas instituciones e investigadores, la situación de los archivos municipales de la antigua provincia zoque y de la gran mayoría de los municipios del estado de Chiapas, requiere de estrategias para rescatar y mejorar esta situación, además de acciones que promuevan la obligación de la transparencia y el respeto al derecho de acceso a la información de parte de las autoridades municipales.

\section{El proyecto de "Rescate de archivos históricos de la provincia zoque (siglos XVI al XXI)"}

En este contexto la Universidad de Ciencias y Artes de Chiapas (Unicach) encabezó el proyecto de "Rescate de archivos históricos de la provincia zoque (siglos XVI al XXI)", con el objetivo de realizar un diagnóstico de los archivos municipales ubicados en la antigua provincia zoque del estado de Chiapas, para poder diseñar propuestas enfocadas a la conservación de los archivos históricos municipales y brindar el apoyo necesario tanto a las autoridades como a los distintos sectores de la sociedad civil.

El proyecto surgió a fines del año 2011 cuando los investigadores Sergio Nicolás Gutiérrez Cruz y Fermín Ledesma Domínguez se interesaron en la búsqueda de información documental sobre las haciendas de la antigua provincia zoque, por lo cual la coordinación de la licenciatura en historia de la Unicach diseñó una propuesta de trabajo para la realización de este proyecto, contando con la colaboración de Apoyo al Desarrollo de Archivos y Bibliotecas de México, asociación civil (ADABI A. C.). Por lo que este diagnóstico representó un esfuerzo conjunto de la Unicach y ADABI para contribuir en el rescate y conservación de los archivos municipales de la antigua provincia zoque, con el objetivo de preservar el patrimonio documental y cultural del estado de Chiapas. 


\section{Metodología para el diagnóstico de los archivos municipales}

\section{Organización del trabajo}

A fines de 2011 y principios de 2012 se realizaron reuniones de trabajo encabezadas por la coordinación de la licenciatura en historia, con el propósito de definir las acciones y los objetivos del proyecto para la ejecución de un censo diagnóstico en los municipios ubicados en las región histórica de los zoques, delimitando el área de estudio en 25 municipios: Amatán, Berriozábal, Chapultenango, Chicoasén, Cintalapa, Coapilla, Copainalá, Francisco León, Ixhuatán, Ixtacomitán, Ixtapangajoya, Jiquipilas, Juárez, Ocotepec, Ocozocoautla de Espinosa, Ostuacán, Pantepec, Pichucalco, Rayón, San Fernando, Solosuchiapa, Sunuapa, Tapalapa, Tapilula y Tecpatán.

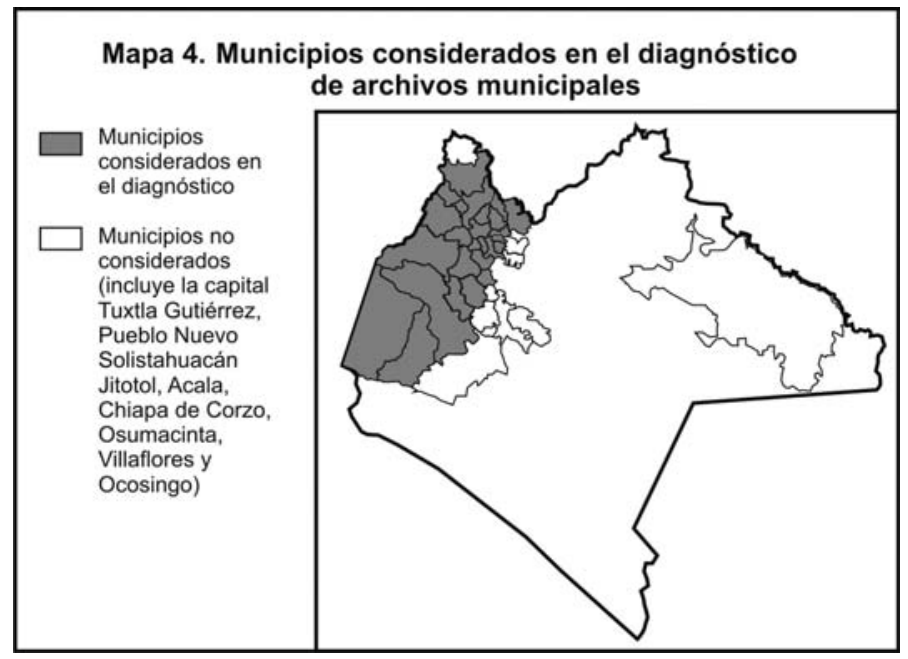

Por la necesidad de obtener la mayor cantidad de datos e información sobre los archivos de la región se decidió realizar el diagnóstico abarcando archivos de diversa índole: de ayuntamientos y agencias municipales, registros civiles y agencias estatales, eclesiásticos o parroquiales, y en menor importancia, archivos de particulares o de propietarios de ranchos y haciendas, por la existencia de información en archivos de la Secretaría de la Reforma Agraria. 


\section{Contacto con autoridades}

A principios de 2012 se realizaron visitas a los municipios de Copainalá, Juárez, Ocotepec, Solosuchiapa, Pichucalco y Tapilula, para llevar a cabo reuniones con autoridades y representantes de los ayuntamientos, agencias municipales, casas de la cultura, oficinas parroquiales, comisariados ejidales y propietarios de ranchos y haciendas, con el objetivo de conocer sus opiniones y sugerencias sobre la ejecución de este proyecto, promover la participación y el interés sobre la conservación de los archivos y tomar en cuenta propuestas o inquietudes acerca de la importancia de preservar la documentación y permitir el acceso a la población.

Algunas autoridades mostraron cierto interés en el proyecto y muchas no contaban con información sobre sus archivos o desconocían totalmente del resguardo de la documentación. Después de varias reuniones las autoridades de los ayuntamientos dijeron no tener conocimiento sobre los archivos de las anteriores administraciones por lo que podrían estar en manos de los expresidentes municipales o probablemente habrían desaparecido al final de cada administración.

Algunos miembros de las casas de la cultura, oficinas parroquiales y propietarios de haciendas ofrecieron su colaboración en la búsqueda de archivos y se interesaron en obtener asistencia para mejorar la conservación y evitar la desaparición de los archivos municipales.

\section{Contacto con archivos}

Además de realizar acercamientos con las autoridades se consultaron archivos de propietarios de ranchos y haciendas de los municipios de Pichucalco y Reforma, y se realizó una visita al archivo del ayuntamiento de Chapultenango, donde se observaron las condiciones y las necesidades para la conservación y ordenación de los documentos. Durante estas visitas los propietarios y autoridades mostraron interés en conservar y permitir el acceso a los participantes del proyecto.

En algunos casos los propietarios de ranchos y fincas estaban dispuestos a donar su documentación a la universidad para permitir su resguardo y facilitar la realización de investigaciones sobre la historia de sus propiedades. Durante la visita al archivo del ayuntamiento de Chapultenango, se pudo constatar la problemática de la preservación de los documentos de las 
administraciones municipales, observando la cantidad, el contenido, el orden y el deterioro de la documentación.

\section{Asesoramiento técnico}

Durante abril y mayo se realizaron reuniones para hacer un balance del proyecto y contar con la asesoría de expertos en rescate de archivos municipales, se mantuvo una estrecha colaboración con el historiador Óscar Janiere Martínez Ruiz, investigador de la Dirección de patrimonio e investigación cultural del Coneculta, quien encabezó el rescate del archivo municipal de Copainalá, que se frustró ante la quema inesperada del archivo. También se contó con el apoyo de los historiadores Víctor Gallardo Zavaleta, encargado del sistema integral de archivos de la Unicach, y de Fabiola Aguilar López, encargada del rescate y ordenación del archivo del ayuntamiento de Comitán. Gracias al apoyo otorgado por estos historiadores con experiencia en el rescate de archivos, se logró trazar las líneas de acción para la realización del diagnóstico en los 25 municipios considerados en el proyecto.

El asesoramiento técnico permitió conocer diversas necesidades y dificultades a tomarse en cuenta, como el contacto y la comunicación con las autoridades y los encargados de los archivos, la búsqueda, la observación y la evaluación de la documentación; la descripción, anotación y recopilación de datos e información sobre el origen, el contexto y la administración de los archivos. Además de la planeación de las visitas, entrevistas y el uso de recursos materiales para la realización del censo diagnóstico en diferentes municipios e instituciones.

\section{Elaboración de cédula de identificación}

Para la realización de las entrevistas con autoridades y encargados de archivos se elaboró una cédula de identificación con el fin de facilitar el diagnóstico de los mismos al momento de visitar los municipios de la región, y así recopilar datos e información sobre el estado de conservación, conocer el contexto y origen de la documentación.

La cédula de identificación se elaboró destacando la información y las características del acervo en seis apartados: 1. información general, 2. condiciones del archivo, 3. tipo de documentación, 4. estado de 
conservación, 5. tipo de ordenación y clasificación, y 6. colecciones y soportes anexos. La elaboración de este instrumento estuvo a cargo del historiador Henry Moguel Villatoro, y se realizó durante los meses de junio y julio en reuniones con investigadores y asesores del proyecto.

\section{Identificación de los archivos}

A partir de los avances y el asesoramiento técnico, se programaron las visitas a los 25 municipios para la realización de los diagnósticos, para este trabajo se capacitó a los egresados de la licenciatura en historia de la Unicach, Luis Nabor Chandoquí Albores, José Levi Morales Manuel, Belsaida Morales Urquín y Josué Santiago Castro, quienes recibieron el apoyo como becarios de este proyecto para acudir individualmente a cuatro diferentes áreas: 1) Amatán, Ixhuatán, Ixtacomitán, Ixtapangajoya, Juárez y Solosuchiapa; 2) Chapultenango, Ostuacán, Pichucalco, Rayón, Sunuapa y Tapilula; 3) Coapilla, Francisco León, Ocotepec, Pantepec, Tapalapa y Tecpatán; 4) Berriozábal, Chicoasén, Cintalapa, Copainalá, Jiquipilas, Ocozocoautla de Espinosa y San Fernando.

En cada una de las cuatro áreas los becarios del proyecto se entrevistaron con autoridades y representantes de ayuntamientos, agencias municipales, casas de la cultura, oficinas parroquiales y registros civiles para conocer las necesidades, problemáticas y situaciones de los archivos con ayuda de la cédula de identificación. Las visitas y entrevistas con las autoridades de los 25 municipios se realizaron gracias a las contribuciones económicas otorgadas a través del convenio entre la Unicach y ADABI.

\section{Revisión de resultados}

Los diagnósticos realizados por los becarios en su mayoría se trataron de archivos de oficialías del registro civil, le siguieron en menor número los archivos eclesiásticos o parroquiales y en la menor cantidad se pudieron observar los archivos de ayuntamientos. Un factor que repercutió en la realización del diagnóstico fue la celebración de las elecciones federales y estatales del primero de julio del 2012, ya que generó conflictos poselectorales en la elección de presidentes municipales y desencadenó movilizaciones y disputas al interior de los municipios. Además, la entrega y recepción de la administración municipal se llevó a cabo el primero de 
octubre esto ocasionó que las autoridades salientes y entrantes ofrecieran poco interés al diagnóstico de los archivos.

En los diagnósticos de archivos eclesiásticos, los representantes de las parroquias se mostraron preocupados por la conservación de los documentos, y después de la explicación de los objetivos del proyecto, se mostraron bastante accesibles e interesados. En los archivos de oficialías del registro civil, se contó con la disponibilidad y accesibilidad de los encargados, quienes se mostraron interesados y permitieron la observación de los documentos más antiguos de finales del siglo XIX y principios del siglo $\mathrm{xx}$, respetando la protección de datos personales y observando exclusivamente las condiciones físicas de los documentos.

\section{Evaluación de resultados}

La revisión de los resultados estuvo a cargo del historiador Justus Fenner, investigador del Programa de Investigaciones Multidisciplinarias de Mesoamérica y el Sureste de la Universidad Nacional Autónoma de México y de Fabiola Aguilar López, quienes evaluaron los resultados del censo diagnóstico obtenidos mediante las observaciones, los datos y la información recopilada en las entrevistas y las cédulas de identificación.

La evaluación de los resultados permitió agrupar y clasificar la información del censo diagnóstico, examinando la información de tres archivos de ayuntamientos de los municipios de Chapultenango, Rayón y Solosuchiapa; siete archivos eclesiásticos de las parroquias de los municipios de Cintalapa, Copainalá, Jiquipilas, San Fernando, Tapalapa, Tapilula y Tecpatán; y 16 archivos del registro civil de los municipios de Berriozábal, Chicoasén, Coapilla, Copainalá, Ixhuatán, Ixtacomitán, Jiquipilas, Ocotepec, Ocozocoautla de Espinosa, Ostuacán, Rayón, San Fernando, Sunuapa, Tapalapa, Tapilula y Tecpatán. A continuación se presenta el balance final con características de los 26 archivos detectados en 19 municipios de la antigua provincia zoque.

\section{Balance final del diagnóstico de los archivos municipales}

\section{Archivos de ayuntamientos}

De los 25 municipios visitados únicamente se realizaron los diagnósticos 
de tres archivos de ayuntamientos, ya que en algunos municipios las autoridades salientes se encontraban en proceso de cierre de administración, por lo tanto las autoridades no permitieron realizar el diagnóstico de los archivos, tal es el caso de los ayuntamientos de: Amatán, Francisco León, Ixtacomitán, Ixtapangajoya, Pantepec y Tapalapa. En otros casos, las autoridades entrantes no permitieron la detección y diagnóstico de los acervos, a causa de desconocimiento, y cierto desinterés o desconfianza, como en el caso de: Cintalapa, Jiquipilas, Juárez, Ocozocoautla de Espinosa, Ostuacán, Pichucalco y Sunuapa; y en otros, a pesar de que las autoridades mostraron cierto interés y ofrecieron atención al diagnóstico, no se logró encontrar el archivo histórico, como en el caso de: Berriozábal, Chicoasén, Copainalá, Coapilla, Ixhuatán, Ocotepec, San Fernando, Tapilula y Tecpatán.

De los tres archivos diagnosticados, el del ayuntamiento de Rayón es el más extenso y antiguo, pues cuenta con 45 metros lineales aproximadamente, y la documentación más antigua es de 1920; le sigue en extensión y antigüedad el de Chapultenango, con 40 metros lineales y con fecha más antigua de 1979; y con menor documentación y antigüedad se observó el de Solosuchiapa con 24 metros lineales cuya documentación más antigua es de 1989. Los tres se ubicaban en su mayoría desordenados, aunque en algunas partes poseían anotaciones de clasificaciones de expedientes y legajos. Los tres archivos se encontraban seriamente afectados por humedad, polvo y en menor grado ataques de roedores e insectos. Los archivos de Chapultenango y Rayón se ubicaban en el área de oficinas de la presidencia municipal, no así el de Solosuchiapa el cual se encontró en el antiguo mercado público que fungía como bodega de materiales desechados por el ayuntamiento. El mobiliario era insuficiente, ya que en su mayoría la documentación no se encontraba en estantería o rebasaba su capacidad de almacenamiento, manteniendo la documentación apilada sobre el piso, además de no contar con cajas para almacenar los documentos, o incluso se encontraban en muebles improvisados como en el caso de Solosuchiapa donde se usaban ataúdes.

Los encargados de los archivos de Chapultenango y Rayón se mostraron interesados en llevar a cabo el rescate de su documentación, reconociendo la importancia para conocer la historia de su localidad y contar con la documentación ordenada y clasificada para su manejo y consulta. La mayor 
preocupación de los encargados era la del robo de documentos y el desalojo que podrían ordenar las nuevas administraciones ante el desinterés y el abandono. Por un lado el archivo de Chapultenango es importante por el registro de actividades del ayuntamiento durante la erupción del volcán Chichonal de 1982; y por otro, el archivo de Rayón es hasta ahora el archivo con la documentación más antigua: 1920. El archivo de Solosuchiapa también puede representar un buen ejemplo para realizar su rescate, aprovechando que la documentación se ha conservado en el antiguo mercado público.
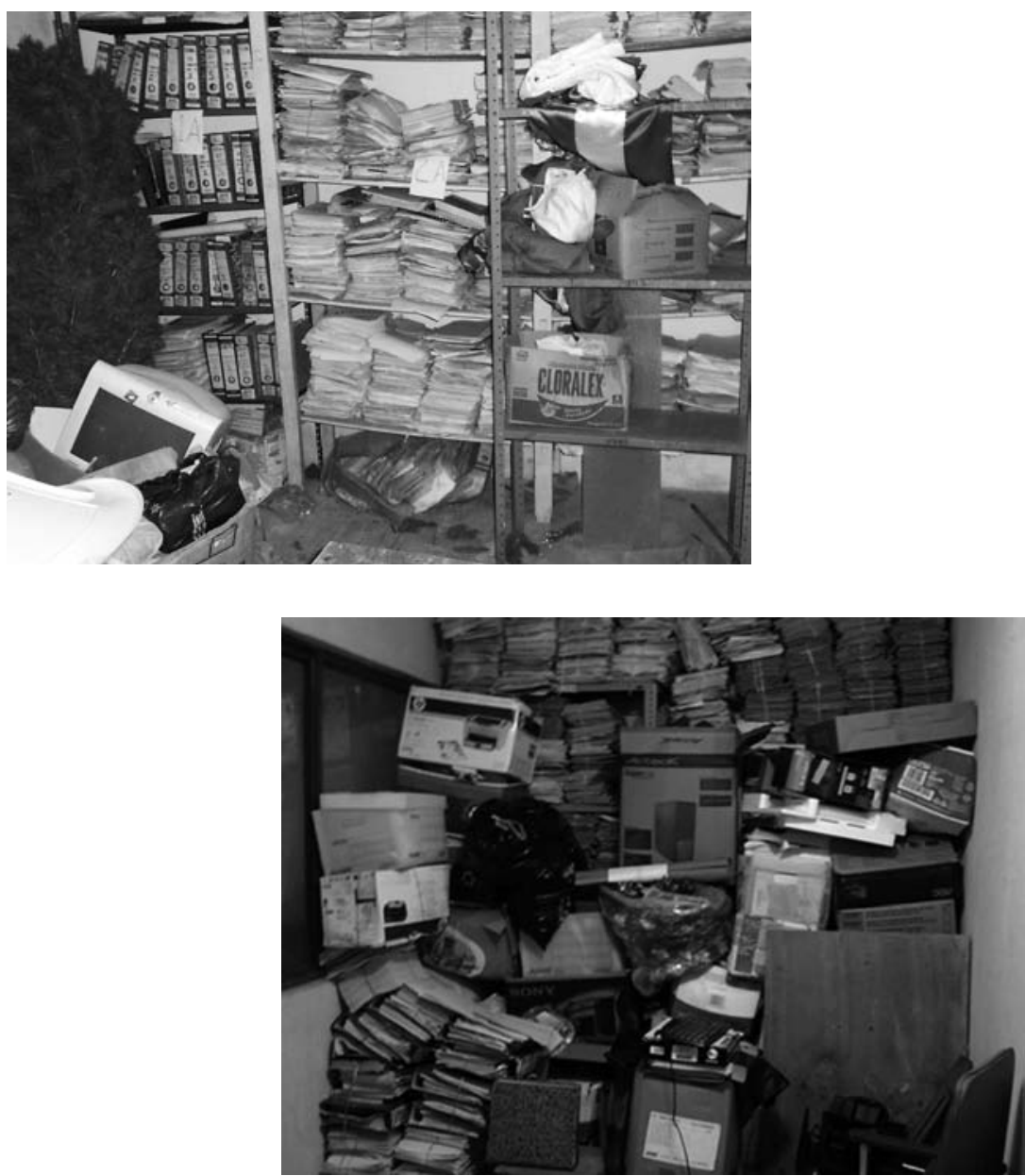


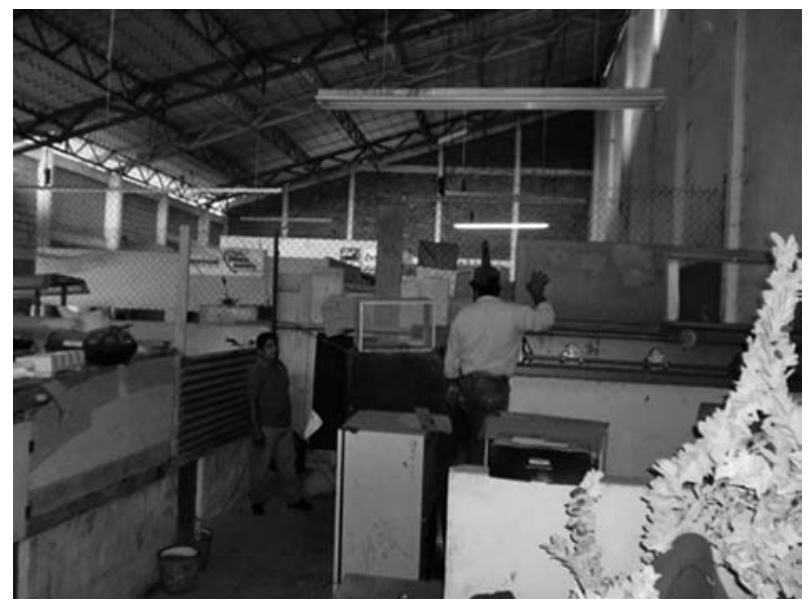

\section{Archivos eclesiásticos}

En el caso de los archivos eclesiásticos se detectaron y diagnosticaron siete archivos históricos, siendo el de Copainalá el más amplio con cinco metros lineales, seguido por los de Cintalapa, Tapilula y Tecpatán con cuatro metros lineales cada uno, y con menor documentación se encontraron los de Jiquipilas, San Fernando y Tapalapa con tres metros lineales cada uno. El de Tecpatán cuenta con la documentación más antigua del año 1627, a continuación se encontraban el de Tapalapa de 1705, Tapilula de 1717, Cintalapa con documentos de 1820, Copainalá de 1887 y los de Jiquipilas y San Fernando con documentos de 1963 y 1996 respectivamente. En su mayoría los archivos contaban con ciertas medidas de prevención para conservar los documentos, aunque también tenían afectaciones por humedad y polvo. Los archivos de Tapalapa y Tapilula poseen información del siglo XVIII, ésta debido a las condiciones climáticas y a la falta de mobiliario presenta en la mayor parte de los documentos afectaciones causadas humedad, polvo, insectos y roedores.

En la mayoría de los municipios no se lograron detectar archivos eclesiásticos por la falta de autorización o la ausencia del párroco, como en los casos de: Amatán, Berriozábal, Chapultenango, Francisco León, Ixhuatán, Ocozocoautla de Espinosa, Pantepec y Solosuchiapa. En el caso de los municipios de Ixtacomitán, Ixtapangajoya, Juárez, Ostuacán, Pichucalco 
y Sunuapa, argumentaron que los archivos habían sido transferidos a la parroquia del municipio de Reforma, ya que en ella se contaba con mayor espacio y mobiliario; al igual que los de la parroquia de Rayón, los cuales fueron transferidos a la sede de la orden de los franciscanos en la ciudad de Izamal en el estado de Yucatán.

\section{Archivos de oficialías del registro civil}

En el caso de los archivos del registro civil se observaron 16 archivos, siendo los de Berriozábal, Jiquipilas, Ocozocoautla y San Fernando los que contaban aproximadamente con más 30 metros lineales de documentación cada uno. En un parámetro intermedio se encontraban los de: Tecpatán con 22, Ocotepec con 20, Copainalá con 25, Tapilula y Ostuacán con 18, Ixhuatán, Ixtacomitán y Rayón con 16 metros lineales. Entre los de menor documentación se encontraban: Tapala con cuatro, Sunuapa con seis, Chicoasén con nueve y Coapilla con 12 metros lineales. Los archivos con documentación más antigua fueron los de San Fernando de 1860, Ocozocoautla de 1861, Berriozábal y Tapilula de 1897 y Copainalá de 1898.

En el diagnóstico se observó que los archivos estaban ordenados, clasificados y puestos a la disposición de los solicitantes de información, además contaban con edificios apropiados para la conservación, aunque en algunos casos no dejaban de tener problemas ocasionados por la humedad y la temperatura. Los documentos más antiguos, del siglo XIX y primeras décadas del siglo Xx, se conservaban en carpetas, sobres o bolsas de plástico. En casos como los de Coapilla, Copainalá, Jiquipilas y Ostuacán requerían de estantería y cajas para evitar que los documentos permanecieran apilados en el suelo.

En algunos municipios no se lograron observar los archivos ante la negativa del oficial del registro civil, como en los casos de: Amatán, Chapultenango, Cintalapa, Francisco León, Ixtapangajoya, Juárez, Pantepec, Pichucalco y Solosuchiapa. 
Tabla 1. Balance final del diagnóstico de ARChivos MUNiCipales

DE LA ANTIGUA PROVINCIA ZOQUE

AÑo DE ANTIGÜEDAD / LONGITUd METROS LINEALES

\begin{tabular}{|c|c|c|c|c|c|c|}
\hline \multirow[t]{2}{*}{ Municipio } & \multicolumn{2}{|c|}{$\begin{array}{c}\text { Archivo de } \\
\text { ayuntamiento }\end{array}$} & \multicolumn{2}{|c|}{ Archivo eclesiástico } & \multicolumn{2}{|c|}{$\begin{array}{c}\text { Archivo de registro } \\
\text { civil }\end{array}$} \\
\hline & Año & MI & Año & MI & Año & MI \\
\hline \multicolumn{7}{|l|}{ 1. Amatán } \\
\hline 2. Berriozábal & & & & & 1897 & 30 \\
\hline 3. Chapultenango & 1979 & 40 & & & & \\
\hline 4. Chicoasén & & & & & 1903 & 9 \\
\hline 5. Cintalapa & & & 1820 & 4 & & \\
\hline 6. Coapilla & & & & & 1918 & 12 \\
\hline 7. Copainalá & & & 1887 & 5 & 1898 & 25 \\
\hline \multicolumn{7}{|l|}{ 8. Francisco León } \\
\hline 9. Ixhuatán & & & & & 1920 & 16 \\
\hline 10. Ixtacomitán & & & & & 1911 & 16 \\
\hline \multicolumn{7}{|l|}{ 11. Ixtapangajoya } \\
\hline 12. Jiquipilas & & & 1963 & 3 & 1917 & 30 \\
\hline \multicolumn{7}{|l|}{ 13. Juárez } \\
\hline 14. Ocotepec & & & & & 1903 & 20 \\
\hline $\begin{array}{l}\text { 15. Ocozocoautla } \\
\text { de E. }\end{array}$ & & & & & 1861 & 30 \\
\hline 16. Ostuacán & & & & & 1915 & 18 \\
\hline \multicolumn{7}{|l|}{ 17. Pantepec } \\
\hline \multicolumn{7}{|l|}{ 18. Pichucalco } \\
\hline 19. Rayón & 1920 & 45 & & & 1916 & 16 \\
\hline 20. San Fernando & & & 1996 & 3 & 1860 & 30 \\
\hline 21. Solosuchiapa & 1989 & 24 & & & & \\
\hline 22. Sunuapa & & & & & 1916 & 6 \\
\hline 23. Tapalapa & & & 1705 & 3 & 1918 & 4 \\
\hline 24. Tapilula & & & 1717 & 4 & 1897 & 18 \\
\hline 25. Tecpatán & & & 1627 & 4 & 1905 & 22 \\
\hline
\end{tabular}




\section{Consideraciones finales}

Entre los resultados del diagnóstico destacó el no tener acceso a los archivos de 22 ayuntamientos, a causa principalmente de desconocer su ubicación o, probablemente, por la situación de abandono en la cual se encuentran, incluso por la desaparición del archivo en anteriores administraciones. El diagnóstico también demostró que el abandono de los archivos se origina especialmente por el desinterés del cabildo y el comité ejecutivo de la presidencia municipal así como por la falta de recursos materiales y capacitación del personal.

En los archivos de los ayuntamientos de Chapultenango, Rayón y Solosuchiapa, se pudo constatar la necesidad de edificios o instalaciones para el acomodo y la conservación del archivo, además de la falta de recursos para mobiliario y unidades de almacenamiento, así como la necesidad de capacitar al personal administrativo del ayuntamiento sobre el manejo y la preservación del archivo; ante estas necesidades y carencias los tres archivos no se encontraban disponibles o abiertos para brindar atención a la ciudadanía.

En los 22 municipios donde no se detectó el archivo del ayuntamiento, no se descarta la existencia de documentación histórica en alguna bodega o instalación del ayuntamiento municipal, como es el caso de Copainalá cuya información se encontró en una bodega a las afueras de la cabecera municipal, o como el de Solosuchiapa la cual ha permanecido en el antiguo mercado público durante más de 10 años. Por esta razón, las autoridades municipales que ingresan en cada trienio no cuentan con información sobre la ubicación de los archivos de las anteriores administraciones, y reaccionan con apatía y desinterés ante la imposibilidad de atender la solicitud de acceso a los archivos.

Hasta la fecha el estado de Chiapas cuenta con pocos archivos de ayuntamientos abiertos regularmente, la situación observada en este diagnóstico puede tomarse como una referencia sobre la situación que presentan los 123 municipios del estado de Chiapas. En los últimos 10 años tan sólo se tiene conocimiento de la existencia de los archivos de los ayuntamientos de San Cristóbal y Comitán, donde a base de esfuerzos la sociedad civil ha solicitado la atención del cabildo y el comité ejecutivo del ayuntamiento para conservar el propio archivo del gobierno municipal. 
Los esfuerzos que hasta ahora se han llevado a cabo por investigadores, como los realizados por Jose Alejos en el archivo del ayuntamiento de Tumbalá, por Justus Fenner en la región de los Altos de Chiapas, y por Carlos Román en la región del Soconusco en la costa del Pacífico, han consistido únicamente en el rescate temporal de documentos sin llegar a la administración y conservación de los archivos de forma permanente. ${ }^{11}$ Esta situación se debe a que las autoridades de los ayuntamientos no se sienten obligadas por las leyes ni por autoridades superiores, como tampoco atienden la obligación de respetar el derecho al acceso a la información y rendir cuentas a la ciudadanía.

El gobierno del estado a través de la creación del Instituto de Acceso a la Información Pública para el estado de Chiapas (IAIP) en 2006, comenzó a promover la transparencia y el acceso a la información en las autoridades del nivel estatal, obligándolas por ley a crear sus portales de transparencia y a brindar atención a las solicitudes de la ciudadanía, sin embargo, las autoridades del nivel municipal continúan sin atender las obligaciones promovidas a través de este instituto, y sin interesarse en la importancia de la administración y conservación de sus archivos históricos.

Podría significar un gran avance en materia de transparencia y acceso a la información si la labor del IAIP trascendiera hacia el nivel municipal, promoviendo en las autoridades el respeto a este derecho y motivando a que el Congreso del Estado otorgue recursos destinados exclusivamente a la creación de los archivos en cada ayuntamiento, además de la contratación o capacitación del personal para su manejo y atención.

Los archivos históricos además de ser un espacio para que la ciudadanía ejerza su derecho al acceso a la información en el ámbito local, deberían ser entendidos tanto por la sociedad como por la clase política, como órganos que garantizan y promueven la rendición de cuentas y la transparencia de la información, que contribuyen a evitar actos de corrupción y por ello

11 Algunos informes sobre las labores del rescate de archivos municipales en Chiapas, han sido publicadas por Alejos, El archivo histórico de Tumbalá; por Román, El Soconusco cervantino; y por Fenner en los artículos: "Recuperación del patrimonio documental de Chiapas", "Fuentes primarias para el Chiapas decimonónico", "Archivos municipales en México y Chiapas" y "El archivo histórico del centro coordinador tzeltal-tzotzil de Chiapas", además del portal en internet de Los Archivos Históricos de Chiapas apoyado por Palomo y el Catálogo del Juggado de Primera Instancia junto con Garza. 
también coadyuvan a que la administración pública sea a favor del interés general de la sociedad.

El diagnóstico de los archivos municipales de la antigua provincia zoque, ha brindado la posibilidad de conocer una inmensa gama de oportunidades no sólo para la Unicach y ADABI, sino para muchos interesados en la historia de la población zoque de Chiapas, y también para aquellos quienes cuentan en sus municipios y en sus instituciones la posibilidad de contribuir, a través de la conservación y administración de los archivos, en el rescate de la historia de Chiapas y en el desempeño democrático de los gobiernos locales de la época actual.

\section{Bibliografía}

Alejos, José y Ortega, Elsa, El archivo municipal de Tumbalá, Chiapas: 19201946. Rescate y conservación de un acervo histórico regional, México, D. F., Centro de Estudios Mayas del Instituto de Investigaciones Filológicas de la Universidad Nacional Autónoma de México, 1990.

Alonso, Marina, "Los zoques bajo el volcán. Microhistoria de la erupción del Chichonal, Chiapas”, tesis que para optar por el grado en doctor en historia, México, D. F., Centro de Estudios Históricos de El Colegio de México, 2011. [Versión electrónica: http://es.scribd.com/ doc/60148295/Alonso-Los-zoques-bajo-el-volcan-Microhistorias-

bajo-la-erupcion-de-El-Chichonal-Chiapas (Consultado el 11/ julio/2014)].

Aramoni, Dolores, Los refugios de lo sagrado. Religiosidad, conflicto y resistencia entre los zoques de Chiapas, México, D. F., Consejo Nacional para la Cultura y las Artes, 1992.

Fenner, Justus, et al., "Recuperación del patrimonio histórico documental de Chiapas: El municipio de San Cristóbal”, en Pueblos y Fronteras, núm. 1, año 2001, San Cristóbal de las Casas, Chiapas, pp. 199-207. [Versión electrónica: http://www.pueblosyfronteras.unam.mx/a01n1/ pdfs/11_recuperacion.pdf (Consultado el 11/julio/2014)].

Fenner, Justus, "Fuentes primarias para el Chiapas decimonónico", en Olivera, Mercedes y Palomo, María Dolores (coords.), Chiapas: de la Independencia a la Revolución, México, D. F./ Tuxtla Gutiérrez, Chiapas, 
Centro de Investigaciones y Estudios Superiores en Antropología Social/ Consejo de Ciencia y Tecnología del Estado de Chiapas, 2005, pp. 385-442. [Versión electrónica: https://www.h-net.org/ latam/ archives/archivo-chiapas.pdf (Consultado el 11/julio/2014)].

, "Archivos municipales en México y Chiapas. Propuestas estratégicas para su rescate, conservación y difusión", en Boletín del Archivo General de la Nación, núm. 2, noviembre-diciembre 2003, México, D. F., pp. 169-177.

y Palomo Infante, Dolores, Los Archivos Históricos de Chiapas. Portal en internet abierto desde el año 2003 del Programa de Investigaciones Multidisciplinarias de Mesoamérica y el Sureste del Instituto de Investigaciones Antropológicas de la Universidad Nacional Autónoma de México con el apoyo del Centro de Investigaciones y Estudios Superiores en Antropología Social-Unidad Sureste, ambos con sede en San Cristóbal de Las Casas, Chiapas. [Versión electrónica:http:/ / www.difusionculturalchiapas.unam.mx/archiapas/arch_hist_chis.html (Consultado el 11/julio/2014)].

"El archivo histórico del centro coordinador tzeltaltzotzil de Chiapas. Memoria del laboratorio del indigenismo en México", en Desacatos, No. 26, enero-abril 2008, México D. F., pp. 75-86. [Versión electrónica: http://www.redalyc.org/pdf/139/13902606.pdf (Consultado el 11/julio/2014)].

y Garza Caligaris, Anna María, Catálogo del Juzgado de $1^{a}$ Instancia de lo Civil, San Cristóbal de Las Casas, Chiapas, 17981920. Portal en internet abierto desde el año 2013 del Programa de Investigaciones Multidisciplinarias de Mesoamérica y el Sureste del Instituto de Investigaciones Antropológicas de la Universidad Nacional Autónoma de México con el apoyo del Instituto de Estudios Indígenas de la Universidad Autónoma de Chiapas, ambos con sede en San Cristóbal de Las Casas, Chiapas. [Versión electrónica: http://www. difusionculturalchiapas.unam.mx/cjp/index.php. (Consultado el 11/ julio/2014)].

Hidalgo, Enrique, La fiesta del enojo. La tradición oral volcánica de los zoques de Chiapas, Tuxtla Gutiérrez, Chiapas, Universidad de Ciencias y Artes de Chiapas, 2009. 
INEGI, Conteo de población y vivienda 2005. Tabulados básicos. (Chiapas: Lengua indígena). [Versión electrónica: http://www3.inegi.org.mx/sistemas/ tabuladosbasicos $/$ LeerArchivo. aspx?ct $=40124 \& \mathrm{c}=33145 \& \mathrm{~s}=\mathrm{est} \& \mathrm{f}=1$ (Consultado el 11/julio/2014)].

Censo de Población y Vivienda 2010. Tabulados del cuestionario básico. (Chiapas: Lengua indígena). [Versión electrónica: http:// www3.inegi.org.mx/sistemas/tabuladosbasicos/LeerArchivo. aspx?ct $=29184 \& c=27302 \& s=$ est\&f=1 (Consultado el 11/julio/2014)] Lee, Thomas A., "La lingüística histórica y la arqueología de los zoquemixe-popolucas", en 1ª Reunión de Investigadores del Area Zoque, Tecpatán, Chiapas, Centro de Estudios Indígenas de la Universidad Autónoma de Chiapas, 1986, pp. 7-36.

Lisbona, Miguel, Cultura y etnicidad zoque. Ensayos para leer una cultura, Tuxtla Gutiérrez, Chiapas, Consejo Estatal para la Cultura y las Artes de Chiapas, 2000.

, Sacrificio y castigo entre los zoques de Chiapas. Cargos, intercambios y enredos étnicos en Tapilula, San Cristóbal de Las Casas, Chiapas, Programa de Investigaciones Multidisciplinarias de Mesoamérica y el Sureste del Instituto de Investigaciones Antropológicas de la Universidad Nacional Autónoma de México, 2004.

, "Olvidados del neozapatismo: los zoques chiapanecos", en Estudios Sociológicos, núm. 2, Vol. xxIv, mayo-agosto 2006, México, D. F., pp. 305-330. [Versión electrónica: http://www.redalyc.org/ pdf/598/59807102.pdf (Consultado el 11/julio/2014)].

Ortiz, Rocío, Lengua e Historia entre Los Zoques de Chiapas. Castellanización, desplazamiento y permanencia de la lengua zoque en la Vertiente del Mezcalapay el Corazón Zoque de Chiapas, Zamora, Michoacán, El Colegio de Michoacán/ Universidad de Ciencias y Artes de Chiapas, 2012.

Reyes, Laureano, Los zoques del volcán, México, D. F., Comisión Nacional para el Desarrollo de los Pueblos Indígenas, 2007.

Román, Carlos, El Soconusco cervantino: Cartografía de una encomienda imaginaria, (Disco compacto interactivo que incluye un catálogo de 340 imágenes), México, D. F., Archivo General de la Nación con el apoyo del Ministerio de Cultura de España, 2005. 
Thomas, Norman D., "Los zoques”, en Esponda, Víctor Manuel (comp.), Lapoblación indígena de Chiapas, Gobierno del Estado de Chiapas/Consejo Estatal para el Fomento a la Investigación y Difusión de la Cultura, Tuxtla Gutiérrez, Chiapas, 1993, pp. 49-90.

Villa Rojas, Alfonso, et al., Los zoques de Chiapas, México D. F., Instituto Nacional Indigenista, 1975.

Viqueira, Juan Pedro, Indios rebeldes e idólatras. Dos ensayos sobre la rebelión india de Cancuc, Chiapas, acaecida en el año de 1712, México, D. F., Centro de Investigaciones y Estudios Superiores en Antropología Social, 1997. "Auge y decadencia de las montañas zoques", en Anuario del Instituto de Estudios Indígenas de la Universidad Autónoma de Chiapas, vol. IX, 2003, Tuxtla Gutiérrez, Chiapas, pp. 341-441. 